\title{
Identification of lymphatic metastasis-associated genes in a metastatic ovarian cancer cell line
}

\author{
YU KANG ${ }^{1-3}$, TAO PU ${ }^{2}$, QINGQING CAI ${ }^{3}$, SHANSHAN HONG ${ }^{2}$, \\ MINGXING ZHANG ${ }^{2}$, GUILING LI ${ }^{1,2}$, ZHILING ZHU ${ }^{1,2}$ and CONGJIAN XU ${ }^{1-3}$ \\ ${ }^{1}$ Department of Integrated Traditional Chinese and Western Medicine, Obstetrics and Gynecology Hospital, Fudan University, \\ Shanghai 200011; ${ }^{2}$ Department of Obstetrics and Gynecology of Shanghai Medical School, Fudan University, \\ Shanghai 200032; ${ }^{3}$ Shanghai Key Laboratory of Female Reproductive Endocrine Related Diseases, \\ Shanghai 200011, P.R. China
}

Received July 15, 2014; Accepted March 24, 2015

DOI: $10.3892 / \mathrm{mmr} .2015 .3743$

\begin{abstract}
Ovarian cancer (OC) is the gynecological malignancy with the highest rate of mortality, and lymphatic metastasis is a critical factor in disease recurrence and prognosis. In the current study, the SKOV-3/LN403 cell line, which has high potential for lymph node metastasis was established as a result of four rounds of selection from retroperitoneal lymph nodes following intraperitoneal injections of SKOV-3 ovarian adenocarcinoma cells. In comparison to the parental SKOV-3 cell line, SKOV-3/LN403 has a higher rate of proliferation, is more invasive and exhibits greater resistance to paclitaxel. Subsequently, a novel animal model of OC lymphatic metastasis was developed with SKOV-3/LN403 cells and a high incidence of positive metastatic lymph nodes, peritoneal dissemination and bloody ascites were observed, which mimicked the clinical outcome of patients with OC. Analysis of the gene expression profiles of SKOV-3 and SKOV-3/LN403 cells identified several genes and pathways that may be involved in lymphatic metastasis of $\mathrm{OC}$. The induction of focal adhesion kinase expression provides a potential therapeutic target for OC lymphatic metastasis.
\end{abstract}

\section{Introduction}

Ovarian cancer (OC) is the gynecological cancer with the highest mortality rate. There were an estimated 22,280 new

Correspondence to: Dr Zhiling Zhu or Dr Congjian Xu, Department of Integrated Traditional Chinese and Western Medicine, Obstetrics and Gynecology Hospital, Fudan University, 419 Fang-Xie Road, Shanghai 200011, P.R. China

E-mail: zhilingzhu888@163.com

E-mail: xucj@hotmail.com

Key words: ovarian cancer, lymph node metastasis, mouse model, focal adhesion kinase cases and 15,500 fatalities in the United States in 2012 and a survival rate of $\sim 44 \%$ (1).

OC metastases include transcoelomic, lymphogenous and hematogenous spread (2). Lymphogenous metastasis is an indicator of disease aggressiveness, recurrence and prognosis $(3,4)$. Kleppe et al (5) reported a $14.2 \%$ incidence of lymphatic metastases in stage I-II epithelial OC, predominantly in the para-aortic and pelvic regions. In advanced-stage disease, lymphatic metastases exceeds $50 \%$ (3,6-9). Preclinical mouse models of lymphatic metastasis in OC remain limited and the majority of models do not mimic the clinical disease (10-14).

In the present study, a novel epithelial OC cell line SKOV-3/LN403 with high metastatic potential for lymph node metastasis was established by selection from the parental SKOV-3 cell line. The biological characteristics and potential genes and pathways associated with the cell line were further investigated. Microarray analysis was used to screen SKOV-3 and SKOV-3/LN403 for genes and pathways involved in the molecular mechanism of lymphatic metastasis.

\section{Materials and methods}

Cell culture and experimental animals. The SKOV-3 human ovarian adenocarcinoma cell line was obtained from the Shanghai Institute of Cell Biology of Chinese Academy of Sciences (Shanghai, China). Cells were grown in RPMI 1640 medium supplemented with $10 \%$ (v/v) fetal bovine serum, $100 \mathrm{U} / \mathrm{ml}$ penicillin and $100 \mathrm{mg} / \mathrm{ml}$ streptomycin (all purchased from Gibco Life Technologies, Grand Island, NY, USA). They were maintained at $37^{\circ} \mathrm{C}$ in a humidified incubator containing $5 \% \mathrm{CO}_{2}$ and cultures were split twice per week.

Six-week-old female BALB/c nude mice were purchased from the Animal Center of the Chinese Academy of Sciences (Shanghai, China) and were maintained under specific pathogen-free conditions. The current study was performed with the approval of the Animal Ethics Committee of the Obstetrics and Gynecology Hospital, Fudan University (Shanghai, China) and in accordance with the Guide for the Ethical Treatment of Laboratory Animals from the Ministry of Science and Technology of the People's Republic of China (publication no. 2006-398) (15). 
Establishment of a novel cell subline. SKOV-3/LN403 was selected following four rounds of the following procedure: SKOV-3 cells were detached with $0.25 \%$ trypsin (Gibco Life Technologies) and resuspended at a density of $1 \times 10^{7}$ cells $/ \mathrm{ml}$. The cells were implanted into the abdominal cavities of five $\mathrm{BALB} / \mathrm{c}$ nude mice by intraperitoneal injection $\left(2 \times 10^{6} /\right.$ mouse $)$. Three weeks later, the mice were sacrificed via cervical dislocation and all visible retroperitoneal and para-aortic lymph nodes were removed. The nodes were then grouped according to the mouse (designated 101-105). Lymph nodes were trypsinized and cultivated under the conditions described above, but with one passage every 3 days. Once stable populations of SKOV-3/LN(101-105) were established, the suspended cells were randomly selected and re-inoculated into five new recipient BALB/c nude mice (mice 201-205). Following the same procedures, SKOV-3/LN(201-205) populations were established and re-inoculated. Following four rounds of selection from the parental SKOV-3 cell line, SKOV-3/LN401-SKOV-3/LN405 were obtained. SKOV-3/LN403 was selected at random and used in the following experiments.

In vitro cell growth assay. SKOV-3 and SKOV-3/LN403 cells were seeded into eight parallel wells of a quantitative cell analyzer (RTCA DP, Roche Diagnostics GmbH, Mannheim, Germany) at a concentration of $1 \times 10^{5}$ cells $/ \mathrm{ml}$ and were observed using phase-contrast microscopy. Each well was seeded with $200 \mu \mathrm{l}$ cells and the cell index was measured over $96 \mathrm{~h}$. Data were analyzed using RTCA software, version 1.2 (ACEA Biosciences Inc., San Diego, CA, USA).

Cell migration assay. Serum-starved SKOV-3 or SKOV-3/LN403 cells were suspended in serum-free medium (SFM) and plated at a density of $1 \times 10^{5}$ cells/well onto gelatin-soaked membranes (Osmonics, Inc., Livermore, CA, USA) in a membrane invasion culture system $(16,17)$. SFM was used as a chemoattractant in the lower wells. Following incubation for $6 \mathrm{~h}$ in RPMI-1640 at $37^{\circ} \mathrm{C}$, the lower chambers were treated with $0.1 \%$ EDTA to retrieve the cells. Randomly selected fields of fixed and stained migrating cells were quantified with Image $\mathbf{J}$ software version 1.48 (National Institutes of Health, Bethesda, MD, USA) (18). Experiments were performed in triplicate.

Cell invasion assay. The membrane invasion culture system was also used to perform invasion assays. In brief, $1 \times 10^{5}$ viable serum-starved SKOV-3 or SKOV-3/LN403 cells were placed onto the upper wells of a defined basement membrane matrix consisting of human laminin (Sigma-Aldrich, St. Louis, MO, USA), type IV collagen (Sigma-Aldrich) and gelatin (MP Biomedicals LLC, Santa Ana, CA, USA) as the barrier to invasion. SFM was used as the chemoattractant in the lower wells. The chambers were incubated for $24 \mathrm{~h}$ at $37^{\circ} \mathrm{C}$ and analyzed as described for the migration assay. Invasion assays were performed in triplicate.

Lymph node metastases in vivo. SKOV-3 and SKOV-3/LN403 cells in the logarithmic phase were harvested and injected into the abdominal cavities of $10 \mathrm{BALB} / \mathrm{c}$ nude mice $\left(2 \times 10^{6}\right.$ cells/mouse). Mouse health, the degree of ascites and survival were observed every 2 days. Following spontaneous death, the mice were dissected and the lymph nodes and organ metastases were examined. The lymphatic nodes were fixed in $10 \%$ neutral formalin, embedded in paraffin, cut into $5 \mu \mathrm{m}$ tissue sections and stained with hematoxylin and eosin (HE). The HE images were captured on an Olympus BX53 microscope (Olympus, Tokyo, Japan) and cellSens Standard software (Olympus).

SPECT-CT imaging in vivo. Small animal SPECT-CT (Nano SPECT/CT PLUS; Bioscan, Inc., Washington, DC, USA) was used to analyze the lymph nodes of an independent group of BALB/c nude mice anesthetized in an anesthetic chamber with $4 \%$ isoflurane (Abbott Laboratories, Chicago, IL, USA) to induce anesthesia, which was maintained with $2 \%$ isoflurane. Each mouse was confined in a gantry and injected with $0.1 \mathrm{mCi}^{99 \mathrm{~m}}$ Tc-sulfur colloid (Beijing Syncor Star Medicinal Technology Co., Ltd., Beijing, China ) in one footpad; although the recommended concentration is $0.3 \mathrm{mCi}$, a smaller concentration was used due to experimental limitations (19). Subsequent to injection, the mice were forced to exercise in the animal cage for $1 \mathrm{~h}$, then were examined by NanoSPECT/CT small animal imaging tomographic $\gamma$-camera for $10 \mathrm{~min}$ with static views. Images were analyzed with in vivo Scope software version 1.44 (Bioscan, Inc.). SPECT-CT imaging was performed at the Department of Nuclear Medicine, Fudan University Shanghai Cancer Center (Shanghai, China).

Drug cytotoxicity assay. The $\mathrm{IC}_{50}$ was determined as described previously (20). Briefly, 3,000 cells were seeded in each well of a 96-well plate and allowed to adhere overnight. Following a resting period of $24 \mathrm{~h}$, the medium was exchanged for one containing increasing concentrations of paclitaxel (Sigma-Aldrich). Following $72 \mathrm{~h}$, a 3-(4,5-dimethylthiazol-2-yl)-2,5-diphenyltetrazolium bromide (MTT) assay was conducted. Absorbance was measured at $570 \mathrm{~nm}$ using a multiwell scanning spectrophotometer (Multiskan; Spectrum, Thermo Electron Co., Vantaa, Finland).

Reverse transcription quantitative polymerase chain reaction (RT-qPCR) analysis. RT-qPCR was performed to assess FAK mRNA expression in SKOV-3 and SKOV-3/LN403 cells using the RNAqueous kit (Ambion, Austin, TX, USA), following the manufacturer's instructions. The mRNA was then reverse-transcribed into cDNA using the Verso cDNA synthesis kit (Thermo Scientific, Pittsburgh, PA, USA). The cDNA was subjected to PCR amplification using the following primers (Sangon Biotech, Shanghai, China): FAK, sense 5'-CCAGGGATTATGAGATTC-3' and antisense 5'-GACAC CAGAACATTCCGAGCA-3' in the Applied Biosystems 7500 system using SYBR Green master mix (Applied Biosystems, Foster City, CA, USA). The amplification reactions were performed for 35 cycles at $94^{\circ} \mathrm{C}$ for $50 \mathrm{sec}, 55^{\circ} \mathrm{C}$ for $30 \mathrm{sec}$ and $72^{\circ} \mathrm{C}$ for $2 \mathrm{~min}$. The products were electrophoresed on a $1 \%$ agarose gel (Sigma-Alrdich) containing ethidium bromide (Sigma-Aldrich) and images of the bands were captured under UV light. $\beta$-actin was used as an endogenous control.

Western blot analysis. Cells at $80 \%$ confluence were harvested and lysed in modified radioimmunoprecipitation assay buffer $(50 \mathrm{mmol} / \mathrm{l}$ Tris, $150 \mathrm{mmol} / \mathrm{l} \mathrm{NaCl}, 1 \%$ Triton X-100, 
A

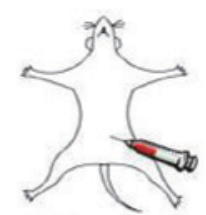

Intraperitoneal injection

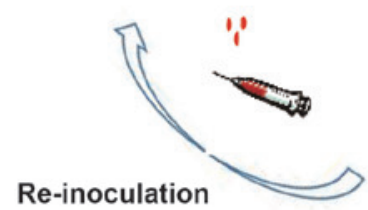

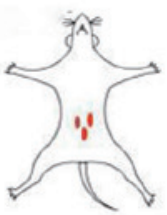

Retroperitoneal lymph node removed

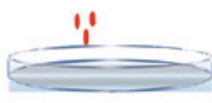

Isolation and Cultivation
B

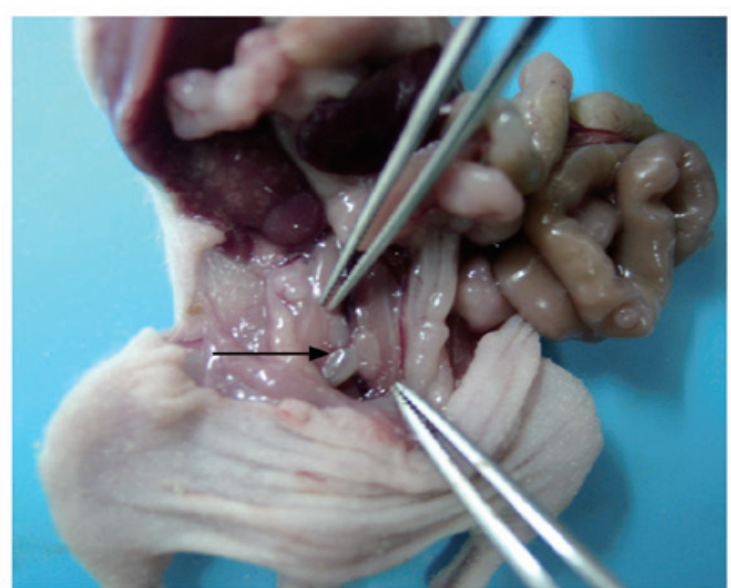

C

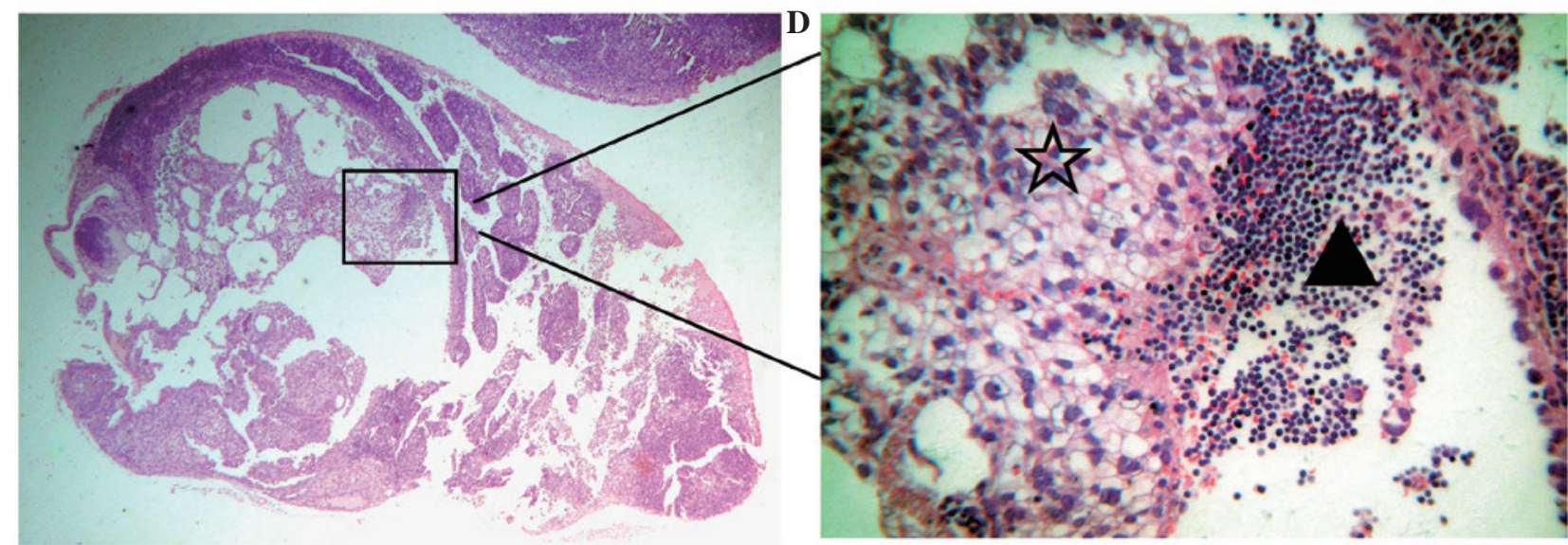

Figure 1. Selection of SKOV-3 and SKOV-3/LN 403 cell lines. (A) The establishment of a highly metastatic subline via in vivo selection. (B) Retroperitoneal lymph node (arrow) in nude mice. Gross anatomy and hematoxylin-eosin staining with a magnification of (C) x40 and (D) x200. Triangle, lymphocytes; star, tumor cells.

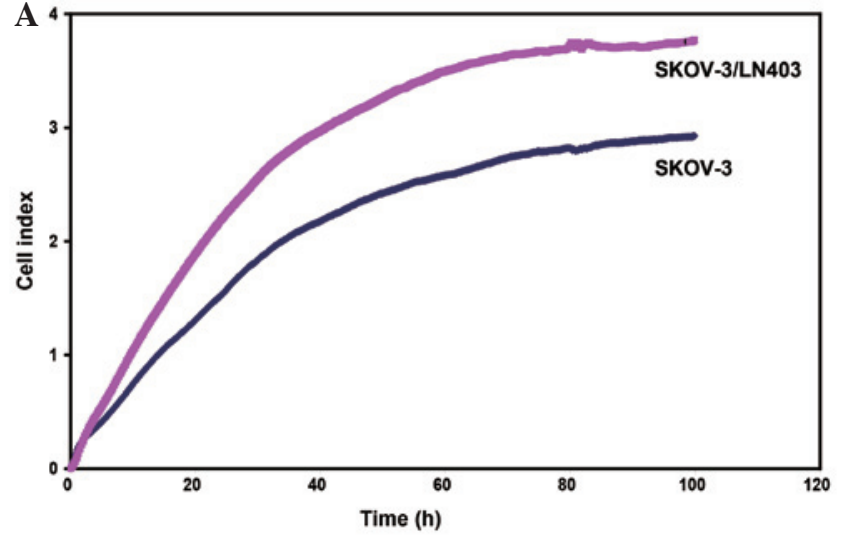

B

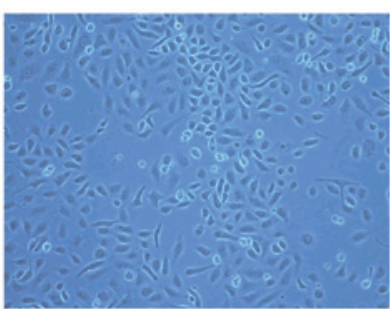

SKOV-3

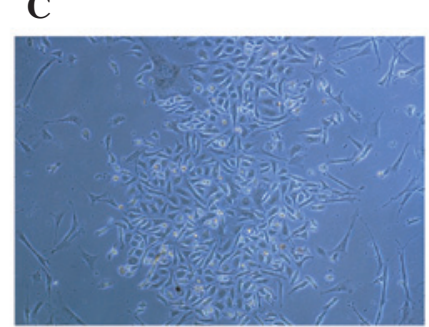

SKOV-3/LN403

Figure 2. Characteristics of SKOV-3 and SKOV-3/LN403 cells. (A) Growth curves of SKOV-3 and SKOV-3/LN403. Morphology as observed by phase-contrast microscopy. (B) SKOV-3 cells had an irregular appearance, while (C) SKOV-3/LN403 grew in clusters; original magnification, x200.

$0.5 \%$ deoxycholate, $25 \mu \mathrm{g} / \mathrm{ml}$ leupeptin, $10 \mu \mathrm{g} / \mathrm{ml}$ aprotinin, $2 \mathrm{mmol} / \mathrm{l}$ EDTA and $1 \mathrm{mmol} / \mathrm{l}$ sodium orthovanadate). Typically, $30 \mu \mathrm{g}$ protein was fractionated by $10 \%$ SDS-PAGE, transferred onto a nitrocellulose membrane (Bio-Rad Laboratories, Inc., Hercules, CA, USA), blocked with 5\% nonfat milk in Tris-buffered saline with Tween-20 (10 mmol/1 Tris (pH 8.0), $150 \mathrm{mmol} / \mathrm{l} \mathrm{NaCl}$ and $0.05 \%$ Tween-20) for $1 \mathrm{~h}$ at ambient temperature, and incubated with primary antibodies against purified mouse anti-FAK (cat. no. 610088; 1:500) and pFAK397 (cat. no. 611807; 1:1,000), each purchased from BD Biosciences (San Jose, CA, USA) at $4^{\circ} \mathrm{C}$ overnight. Antibodies were detected using $0.167 \mu \mathrm{g} / \mathrm{ml}$ horseradish peroxidase-conjugated secondary antibody (Jackson Laboratory, Bar Harbor, ME, USA) and developed using an enhanced chemiluminescence detection kit (Pierce Chemical, Rockford, IL, USA). A densitometric analysis was performed using Image J software (version 1.48) to interpret the differences in western blot results.

cDNA microarray expression analysis and target gene prediction. Total RNA was isolated from SKOV-3 and SKOV-3/LN403 cells with TRIzol (Invitrogen Life Technologies, Carlsbad, CA, USA) and the RNeasy kit (Qiagen, Hilden, Germany) according to the manufacturer's instructions, including a DNase digestion step. RNA quantity and 
A

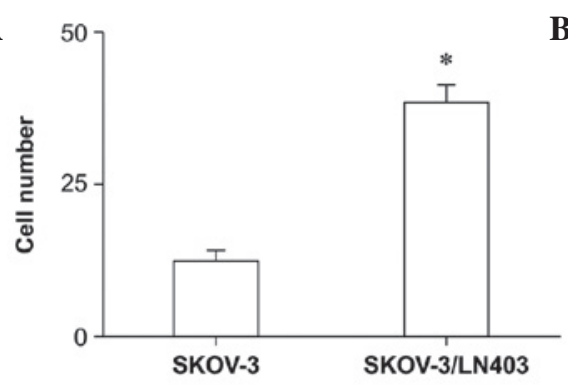

C

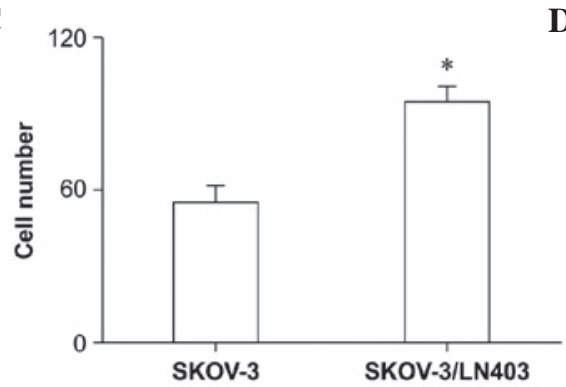

B

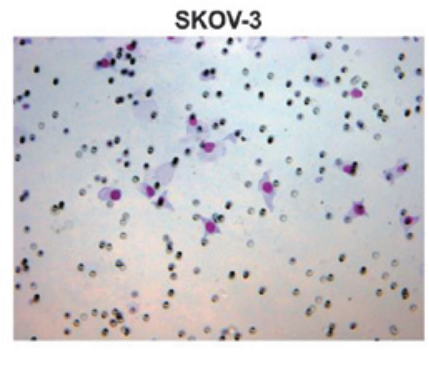

D

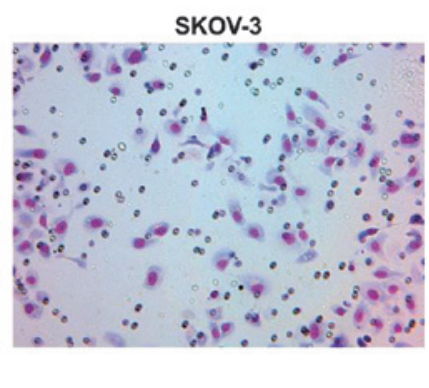

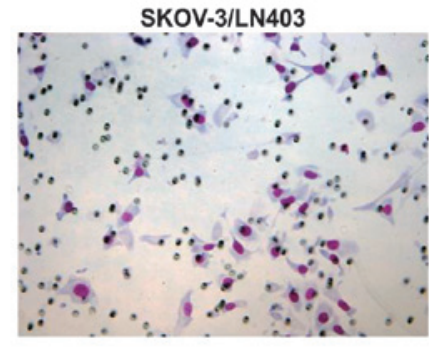

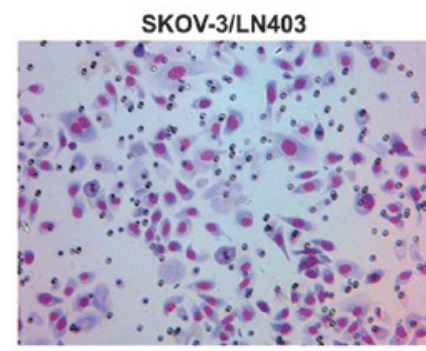

Figure 3. Migration and invasion of SKOV-3 and SKOV-3/LN 403 cells. (A) Average number of attached cells in the migration assay. (B) Representative images of migrating cells. (C) Average number of attached cells in the invasion assay. (D) Representative images of invading cells. Error bars represent the standard error of the mean. ${ }^{*} \mathrm{P}<0.0001$, compared with SKOV-3 cells.

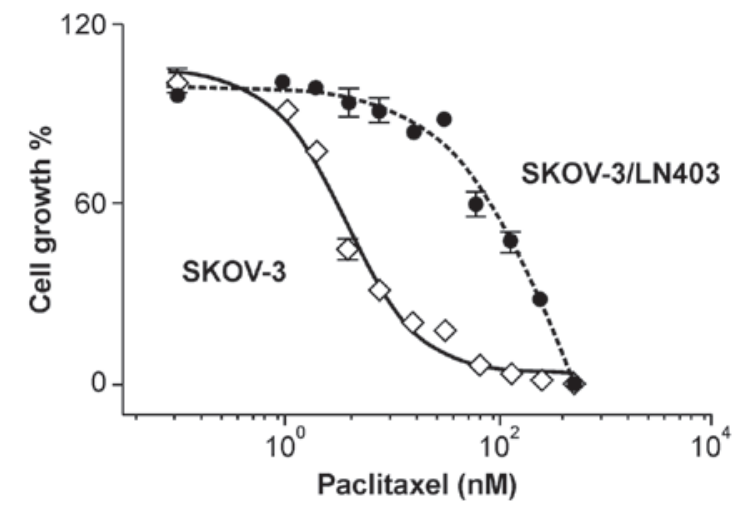

Figure 4. Paclitaxel cytotoxicity. Effect of paclitaxel on the growth of SKOV-3 and SKOV-3/LN403 cells. Each point represents the mean of three independent experiments. Error bars represent the standard error of the mean.

purity were determined using an ND-1000 Spectrophotometer (Thermo Fisher Scientific, Wilmington, DE, USA) and with denaturing gel electrophoresis. The samples were amplified and labeled with the Agilent Quick Amp Labeling kit and hybridized to an Agilent Whole Human Genome Oligo Microarray (Agilent Technologies, Inc., Santa Clara, CA, USA) in Agilent's SureHyb Hybridization Chambers. The array contained 45,000 probes for human genes. Microarray experiments were performed in triplicate according to the manufacturer's instructions. RNA from SKOV-3 and SKOV-3/LN403 cells was reverse-transcribed to cDNA using the Verso cDNA synthesis kit (Thermo Scientific). and labeled with Cy3 and Cy5 dye (GE Healthcare, Chalfont St. Giles, UK), respectively. Hybridization was performed using Agilent's Surehyb chambers at $65^{\circ} \mathrm{C}$ for $16 \mathrm{~h}$. The hybridized slides were washed using Agilent Gene Expression wash buffers (part no. 5188-5327) then scanned with the Agilent DNA microarray scanner using settings recommended by the manufacturer. The microarray datasets were normalized in GeneSpring GX (Agilent Technologies, Inc.) using the Agilent FE one-color scenario (mainly median normalization; Agilent Technologies, Inc.). Fold change (FC) analysis was performed by calculating the ratio of SKOV-3 to SKOV-3/LN403 to identify differentially expressed genes. Genes with an FC $>2$ were identified as upregulated and alterations $<0.5$ fold represented downregulated expression. Functional analysis was performed with the Kyoto Encyclopedia of Genes and Genomes (KEGG) Pathway database (http://www.genome.jp/kegg/pathway. html) using the WEB-based GEne SeT AnaLysis Toolkit (WebGestalt; http://bioinfo.vanderbilt.edu/webgestalt/). The microarray experiment was performed by Shanghai KangChen Biotech, Co., Ltd. (Shanghai, China).

Statistical analysis. Statistical analysis was performed in SPSS, version 10.0 (SPSS, Inc., Chicago, IL, USA) and $\mathrm{P}<0.05$ was considered to indicate a statistically significant difference. All results are expressed as the mean \pm standard error of the mean. Continuous variables were compared using the Student's t-test or an analysis of variance.

\section{Results}

Selection of lymph node-metastatic cells. A flow diagram illustrating the establishment of a highly lymphatic metastatic subline via in vivo selection is presented in Fig. 1A. SKOV-3/LN403 cells were isolated by a series of steps including multiple intraperitoneal injection and continuous screening of the retroperitoneal lymph nodes (Fig. 1B), amplification and in vitro subculture. Hematoxylin-eosin staining illustrated lymphatic node metastasis (Fig. 1C and D).

In vitro grow th curves of SKOV-3 and SKOV-3/LN403 generated by quantitative cell counting demonstrated SKOV-3/LN403 cells to have increased proliferation rates compared with 

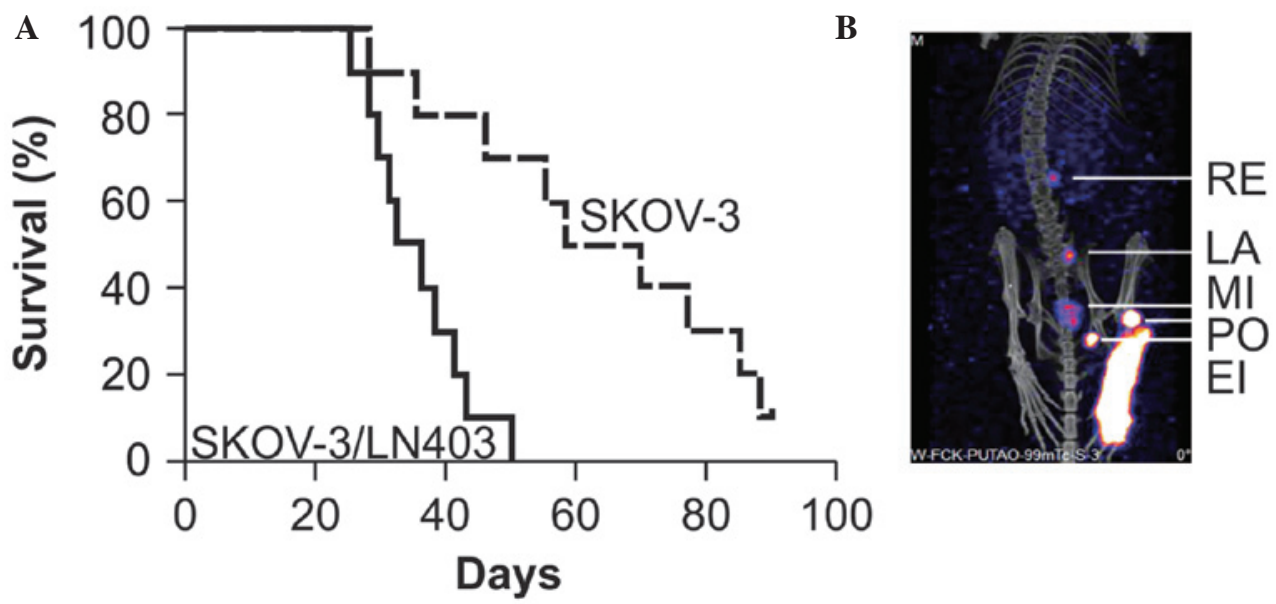

Figure 5. In vivo lymph node metastases of SKOV-3/LN403 cells. (A) Survival curves of nude mice with SKOV-3 and SKOV-3/LN403 cell implants. Mice injected with SKOV-3/LN403 cells had a shorter average survival time of $35.1 \pm 8.3$ days, vs. $64.5 \pm 21.2$ days in mice injected with SKOV-3 cells. (B) Small animal SPECT-CT in an independent group of animals. Drainage lymph nodes including the RE, LA, MI, PO and EI lymph nodes can be clearly visualized. RE, renal; LA, lumbar aortic; MI, medial iliac; PO, popliteal; EI, external iliac.
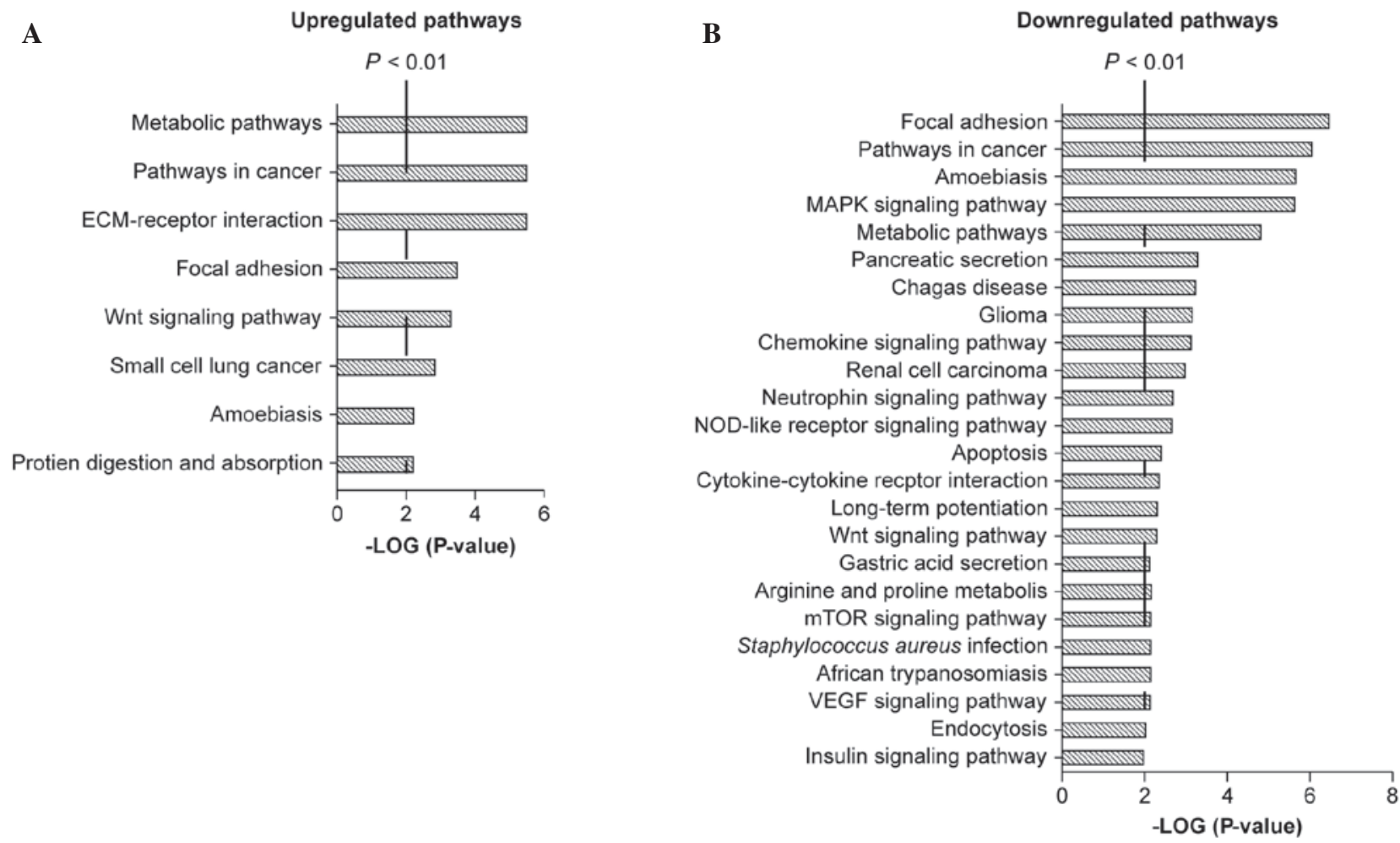

Figure 6. Pathways involved in regulation in SKOV-3 and SKOV-3/LN403 cells. Pathways were identified by WebGestalt, and P<0.01, indicated by the threshold (marked using a dashed line), was considered significant. (A) Upregulated pathways, (B) downregulated pathways.

A

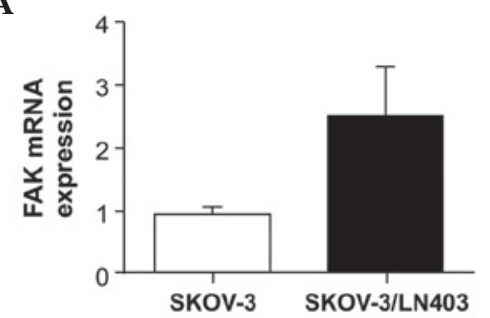

B

pFAK397

FAK

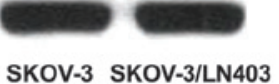

C

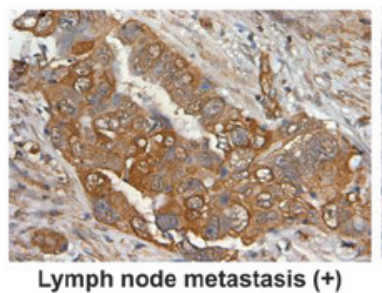

D

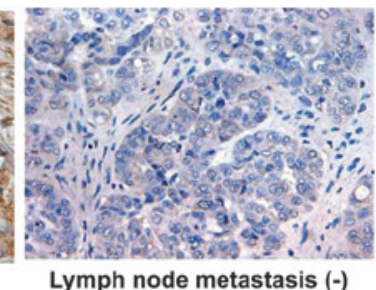

Figure 7. FAK expression in SKOV-3 and SKOV-3/LN403 cells. FAK expression was induced in SKOV-3/LN403 cells: (A) Quantitative polymerase chain reaction; (B) western blotting; and (C and D) immunohistochemical analyses of clinical pathology specimens from three patients with ovarian cancer with/without lymph node metastasis. FAK, focal adhesion kinase. 
SKOV-3 cells. Data was representative of eight independent experiments (Fig. 2A).

The morphological characteristics of SKOV-3 (Fig. 2B) and SKOV-3/LN403 (Fig. 2C) were observed and compared by phase-contrast microscopy. SKOV-3 cells presented an irregular appearance, whilst SKOV-3/LN403 cells grew in clusters.

Cell migration and invasion assay. SKOV-3 and SKOV-3/LN403 cells were seeded and allowed to migrate for $6 \mathrm{~h}$. During this period, $12.40 \pm 1.514$ SKOV-3 cells and $38.50 \pm 2.841 \mathrm{SKOV}-3 / \mathrm{LN} 403$ cells migrated to the lower chamber; this difference was statistically significant (Fig. 3A and B, P<0.0001). For the 24-h invasion assay, the SKOV-3 and SKOV-3/LN403 cell numbers were 55.00 \pm 5.578 and $94.00 \pm 5.455$ (Fig. 3C and $\mathrm{D}, \mathrm{P}<0.0001$ ), respectively, suggesting that SKOV-3/LN403 cells are more invasive.

Drug cytotoxicity assay. The effect of paclitaxel on SKOV-3 and SKOV-3/LN403 cell growth is presented in Fig. 4. The $\mathrm{IC}_{50}$ values of SKOV-3/LN403 and SKOV-3 cells were $104.72 \pm 23.889$ and 5.33 \pm 1.215 nmol, respectively (Fig. 4).

In vivo spontaneous lymphatic metastasis via intraperitoneal injection. The average survival of nude mice with SKOV-3/LN403 cell implants was observed to be $35.1 \pm 8.3$ days The rate of lymph node metastasis was $90 \%$ (9/10), abdominal tumors were widely distributed and grew into an omental cake and bloody ascites were detected in six mice. The average survival time of nude mice with SKOV-3 cell implants was $64.5 \pm 21.2$ days. The rate of lymph node metastasis was $10 \%$ $(1 / 10)$ and bloody ascites were detected in only one mouse (Fig. 5A). Pathological examination identified no metastasis of SKOV-3/LN403 cells to other immune organs (data not shown). In addition, SPECT-CT enabled clear visualization of the drainage lymph nodes (Fig. 5B).

Candidate gene targets in lymphatic metastasis. Microarray analyses of SKOV-3 and SKOV-3/LN403 cells was conducted in order to screen for the genes and pathways involved in the lymphatic metastaticity of the SKOV-3/LN403 cell line. A total of 8 upregulated pathways (Fig. 6A) and 24 downregulated pathways were identified in the KEGG database (Fig. 6B); these included pathways involved in cancer, focal adhesion, metabolism, amebiasis and the Wnt signaling pathway. The results identified differential expression of the focal adhesion pathway components ECM, ITGA, ITGB, RTK and CycD in SKOV-3 and SKOV-3/LN403 cells. Since the focal adhesion kinase (FAK) is a prominent member of the focal adhesion pathway, analysis of FAK expression was undertaken in the two cell lines. RT-qPCR, western blotting and immunohistochemistry identified significant FAK induction in the SKOV-3/LN403 cell line (Fig. 7A and B) and in clinical pathological specimens from three patients with $\mathrm{OC}$ with lymph node metastasis and three patients with OC without metastases (Fig. 7C and D).

\section{Discussion}

An OC animal model was prepared by injecting SKOV-3/LN403 cells into mice and was clinically similar to patients with
OC, with respect to clinical features including: Widespread intra-abdominal metastasis, bloody ascites, up to $90 \%$ lymph node metastasis involving retroperitoneal lymph nodes, significantly shorter average survival times and faster-spreading tumor cells in vivo. The shorter average survival times significantly shortens the experimental cycle, making it convenient to use SKOV-3/LN403 in OC metastasis studies. With reference to data on the anatomy and nomenclature of murine lymph nodes provided by Van den Broeck et al (21), it was observed that SPECT-CT analysis of the BALB/c nude mice used in the current study clearly illustrated the retroperitoneal lymph nodes.

In 1996, Onda et al (22) suggested that the para-aortic lymph nodes and the internal and external iliac and obturator lymph nodes are the most frequent sites of metastasis of ovarian carcinomas. Lymphatic metastasis is a critical factor in cancer staging and prognosis $(23,24)$, thus preclinical evaluation of lymph nodes in small animal models is important. Since the first use of xenografts of human cancer cells into immunodeficient mice in 1969 (25), they have been widely used to explore tumorigenesis and therapeutic efficacy. The existing human OC cell lines HEY, OVCA429, OCC1, OVCAR3, SKOV-3, A2780-s, A2780-cp, OV2008 and ES-2 have been used for tumor transplant models in mice, however, lymph node metastasis seldom occurs in subcutaneous xenograft or abdomen metastasis tumor models with these cell lines in nude mice $(11,12,14,26)$. A2780-cp cells have been reported to metastasize to one or both ovaries; however, regardless of the thickened bursal membrane, the tumor cells were localized inside the ovary and no metastasis occurred (26). The OC high-metastasis strain HD-8910PM was established from lung metastases by $\mathrm{Xu}$ et al (12). Using orthotopic transplantation, Tamada et al (14) established the first model of lymph node metastasis of ovarian carcinomas with the serous-type cell lines MH and KF. The lymph-fixing metastasis cell line SKOV-3-pm ${ }^{3}$, whose lymph metastasis rate can reach up to $100 \%$, was isolated from the SKOV-3 OC cell line with lesser metastatic potential (11). However, in this OC model, SKOV-3-pm ${ }^{3}$ cells were injected into the footpads of nude mice. Unlike human patients with OC, this model does not exhibit abdominal cavity metastasis or ascites.

Using existing SKOV-3 cells, the novel SKOV-3/LN403 cell line was established, which has high lymph node metastasticity, a well-characterized genetic background and stability through repeated subculture. In comparison to the parental SKOV-3 cell line, SKOV-3/LN403 exhibits a higher rate of proliferation and invasion and a greater resistance to paclitaxel.

A total of 8 upregulated pathways and 24 downregulated pathways were identified using the Agilent Whole Human Genome Oligo Microarray. FAK was selected as a target for experimental confirmation. FAK is a non-receptor protein-tyrosine kinase that mediates integrin interaction with the extracellular matrix (27-29). In the context of cancer, FAK is involved in tumor cell growth, migration and invasion, in addition to epithelial-to-mesenchymal transition and angiogenesis $(28,30,31)$. In 1999, overexpression of FAK was first reported in ovarian carcinoma (32). Sood et al (16) evaluated FAK-expressing ovarian cell lines, benign ovarian samples and epithelial OCs, and observed a significant association between FAK overexpression and cancer migration and 
invasion. Several studies also identified cofactors contributing to FAK overexpression (33-36). FAK overexpression has been associated with breast cancer lung metastasis $(37,38)$, and FAK inhibitors have been introduced in novel therapeutic strategies (20,39-41). An association between FAK overexpression and lymphatic metastasis has been identified in non-small-cell lung, oral squamous cell, esophageal, gastric, tongue, laryngeal and endometrial cancer by retrospective analysis of clinical samples (42-49). Upregulation of FAK in the SKOV-3/LN403 cell line and clinical pathological specimens suggests that it may contribute to the high lymphatic metastaticity of the SKOV-3/LN403 cell line. To the best of our knowledge, the current study is the first to report alterations in FAK expression in OC lymphatic metastatic cell lines.

In conclusion, the SKOV-3/LN403 cell line and a novel mouse model of OC lymphatic metastasis were established in the current study. Microarray analysis of SKOV-3 and SKOV-3/LN403 identified several candidate genes and pathways involved in lymphatic metastasis of OC. Induction of FAK expression provides a potential therapeutic target for $\mathrm{OC}$ lymphatic metastasis.

\section{Acknowledgements}

The current study was supported by The National Natural Science Foundation of China (grant no. 81472423); The Shanghai Natural Science Foundation of China (grant no. 13ZR1404300) and The Experimental Animal Special Fund of Science and Technology Commission of Shanghai (grant no. 13140901500).

\section{References}

1. Siegel R, Naishadham D and Jemal A: Cancer statistics, 2012. CA Cancer J Clin 62: 10-29, 2012.

2. Tan DS, Agarwal R and Kaye SB: Mechanisms of transcoelomic metastasis in ovarian cancer. Lancet Oncol 7: 925-934, 2006.

3. di Re F, Baiocchi G, Fontanelli R, Grosso G, Cobellis L, Raspagliesi $\mathrm{F}$ and di Re E: Systematic pelvic and paraaortic lymphadenectomy for advanced ovarian cancer: Prognostic significance of node metastases. Gynecol Oncol 62: 360-365, 1996.

4. Pereira A, Magrina JF, Rey V, Cortes M and Magtibay PM: Pelvic and aortic lymph node metastasis in epithelial ovarian cancer. Gynecol Oncol 105: 604-608, 2007.

5. Kleppe M, Wang T, Van Gorp T, Slangen BF, Kruse AJ and Kruitwagen RF: Lymph node metastasis in stages I and II ovarian cancer: a review. Gynecol Oncol 123: 610-614, 2011.

6. Ayhan A, Gultekin M, Dursun P, Dogan NU, Aksan G, Guven S, Velipasaoglu M and Yuce K: Metastatic lymph node number in epithelial ovarian carcinoma: Does it have any clinical significance? Gynecol Oncol 108: 428-432, 2008.

7. du Bois A, Reuss A, Harter P, Pujade-Lauraine E, Ray-Coquard I, Pfisterer J; Arbeitsgemeinschaft Gynaekologische Onkologie Studiengruppe Ovarialkarzinom; Groupe d'Investigateurs Nationaux pour l'Etude des Cancers Ovariens: Potential role of lymphadenectomy in advanced ovarian cancer: A combined exploratory analysis of three prospectively randomized phase III multicenter trials. J Clin Oncol 28: 1733-1739, 2010.

8. Hacker NF, Valmadre S and Robertson G: Management of retroperitoneal lymph nodes in advanced ovarian cancer. Int J Gynecol Cancer 18 (Suppl 1): 7-10, 2008.

9. Harter P, Gnauert K, Hils R, Lehmann TG, Fisseler-Eckhoff A, Traut A and du Bois A: Pattern and clinical predictors of lymph node metastases in epithelial ovarian cancer. Int J Gynecol Cancer 17: 1238-1244, 2007.

10. House CD, Hernandez L and Annunziata CM: Recent technological advances in using mouse models to study ovarian cancer. Front Oncol 4: 26, 2014.
11. Ruan HY, Li DR, Li L, Guan X and Zhang W: Establishment of human ovarian carcinoma cell lines with directional highly lymphatic metastasis and study of their biological characteristics. Zhonghua Fu Chan Ke Za Zhi 42: 482-486, 2007 (In Chinese).

12. Shenhua X, Lijuan Q, Hanzhou N, Xinghao N, Chihong Z, Gu Z, Weifang D and Yongliang G: Establishment of a highly metastatic human ovarian cancer cell line (HO-8910PM) and its characterization. J Exp Clin Cancer Res 18: 233-239, 1999.

13. Servais EL, Colovos C, Bograd AJ, White J, Sadelain M and Adusumilli PS: Animal models and molecular imaging tools to investigate lymph node metastases. J Mol Med (Berl) 89: 753-769, 2011.

14. Tamada Y, Aoki D, Nozawa S and Irimura T: Model for paraaortic lymph node metastasis produced by orthotopic implantation of ovarian carcinoma cells in athymic nude mice. Eur J Cancer 40: 158-163, 2004.

15. Kang Y, Chen CM and Xu CJ: Secretion of oestrogen from murine-induced pluripotent stem cells co-cultured with ovarian granulosa cells in vitro. Cell Bio Int 35: 871-874, 2011

16. Sood AK, Coffin JE, Schneider GB, Fletcher MS, DeYoung BR, Gruman LM, Gershenson DM, Schaller MD and Hendrix MJ: Biological significance of focal adhesion kinase in ovarian cancer: role in migration and invasion. Am J Pathol 165: 1087-1095, 2004.

17. Sood AK, Bhatty R, Kamat AA, Landen CN, Han L, Thaker PH, Li Y, Gershenson DM, Lutgendorf S and Cole SW: Stress hormone-mediated invasion of ovarian cancer cells. Clin Cancer Res 12: 369-375, 2006.

18. Spannuth WA, Mangala LS, Stone RL, et al: Converging evidence for efficacy from parallel EphB4-targeted approaches in ovarian carcinoma. Mol Cancer Ther 9: 2377-2388, 2010.

19. Khan IU, Shahid A, Ahmad F, Dar UK, Ahmad M and Javed M: Studying the biological feasibility of $\left[{ }^{99} \mathrm{mTc}(\mathrm{CO})_{3}\right]$-dextran-cystein e-cysteine-mannose as a potential molecular radiopharmaceutical for sentinel node detection. Ann Nucl Med 28: 248-256, 2014.

20. Kang Y, Hu W, Ivan C, et al: Role of focal adhesion kinase in regulating YB-1-mediated paclitaxel resistance in ovarian cancer. J Natl Cancer Inst 105: 1485-1495, 2013.

21. Van den Broeck W, Derore A and Simoens P: Anatomy and nomenclature of murine lymph nodes: Descriptive study and nomenclatory standardization in $\mathrm{BALB} / \mathrm{cAnNCrl}$ mice. J Immunol Methods 312: 12-19, 2006.

22. Onda T, Yoshikawa H, Yokota H, Yasugi T and Taketani Y: Assessment of metastases to aortic and pelvic lymph nodes in epithelial ovarian carcinoma. A proposal for essential sites for lymph node biopsy. Cancer 78: 803-808, 1996.

23. Benedet JL, Bender H, Jones H III, Ngan HY and Pecorelli S: FIGO staging classifications and clinical practice guidelines in the management of gynecologic cancers. FIGO Committee on Gynecologic Oncology. Int J Gynaecol Obstet 70: 209-262, 2000.

24. Kim HS, Ju W, Jee BC, Kim YB, Park NH, Song YS, Kim SC, Kang SB and Kim JW: Systematic lymphadenectomy for survival in epithelial ovarian cancer: a meta-analysis. Int J Gynecol Cancer 20: 520-528, 2010.

25. Rygaard $\mathbf{J}$ and Povlsen CO: Heterotransplantation of a human malignant tumour to 'Nude' mice. Acta Pathol Microbiol Scand 77: 758-760, 1969.

26. Shaw TJ, Senterman MK, Dawson K, Crane CA and Vanderhyden BC: Characterization of intraperitoneal, orthotopic and metastatic xenograft models of human ovarian cancer. Mol Ther 10: 1032-1042, 2004.

27. Hanks SK and Polte TR: Signaling through focal adhesion kinase. BioEssays 19: 137-145, 1997.

28. Zhao J and Guan JL: Signal transduction by focal adhesion kinase in cancer. Cancer Metastasis Rev 28: 35-49, 2009.

29. Hanks SK, Ryzhova L, Shin NY and Brábek J: Focal adhesion kinase signaling activities and their implications in the control of cell survival and motility. Front Biosci 8: d982-996, 2003.

30. Schlaepfer DD, Hauck CR and Sieg DJ: Signaling through focal adhesion kinase. Prog Biophys Mol Biol 71: 435-478, 1999.

31. McLean GW, Carragher NO, Avizienyte E, Evans J, Brunton VG and Frame MC: The role of focal-adhesion kinase in cancer-a new therapeutic opportunity. Nat Rev Cancer 5: 505-515, 2005.

32. Judson PL, He X, Cance WG and Van Le L: Overexpression of focal adhesion kinase, a protein tyrosine kinase, in ovarian carcinoma. Cancer 86: 1551-1556, 1999.

33. Gabriel B, Mildenberger S, Weisser CW, Metzger E, Gitsch G, Schüle R and Müller JM: Focal adhesion kinase interacts with the transcriptional coactivator FHL2 and both are overexpressed in epithelial ovarian cancer. Anticancer Res 24: 921-927, 2004. 
34. Grisaru-Granovsky S, Salah Z, Maoz M, Pruss D, Beller U and Bar-Shavit R: Differential expression of protease activated receptor 1 (Parl) and pY397FAK in benign and malignant human ovarian tissue samples. Int J Cancer 113: 372-378, 2005.

35. Shibata K, Kikkawa F, Nawa A, Thant AA, Naruse K, Mizutani S and Hamaguchi M: Both focal adhesion kinase and c-Ras are required for the enhanced matrix metalloproteinase 9 secretion by fibronectin in ovarian cancer cells. Cancer Res 58 : 900-903, 1998

36. Wang X, Urvalek AM, Liu J and Zhao J: Activation of KLF8 transcription by focal adhesion kinase in human ovarian epithelial and cancer cells. J Biol Chem 283 13934-13942, 2008

37. van Nimwegen MJ, Verkoeijen S, van Buren L, Burg D and van de Water B: Requirement for focal adhesion kinase in the early phase of mammary adenocarcinoma lung metastasis formation. Cancer Res 65: 4698-4706, 2005.

38. Mitra SK, Lim ST, Chi A and Schlaepfer DD: Intrinsic focal adhesion kinase activity controls orthotopic breast carcinoma metastasis via the regulation of urokinase plasminogen activator expression in a syngeneic tumor model. Oncogene 25 : 4429-4440, 2006.

39. Halder J, Lin YG, Merritt WM, et al: Therapeutic efficacy of a novel focal adhesion kinase inhibitor TAE226 in ovarian carcinoma. Cancer Res 67: 10976-10983, 2007.

40. Infante JR, Camidge DR, Mileshkin LR, et al: Safety, pharmacokinetic and pharmacodynamic phaseI dose-escalation trial of PF-, an inhibitor of focal adhesion kinase, in advanced solid tumors. J Clin Oncol 30: 1527-1533, 2012.

41. Sun H, Pisle S, Gardner ER and Figg WD: Bioluminescent imaging study: FAK inhibitor, PF-562,271, preclinical study in PC3M-luc-C6 local implant and metastasis xenograft models. Cancer Biol Ther 10: 38-43, 2010.
42. Ji HF, Pang D, Fu SB, Jin Y, Yao L, Qi JP and Bai J: Overexpression of focal adhesion kinase correlates with increased lymph node metastasis and poor prognosis in non-small-cell lung cancer. J Cancer Res Clin Oncol 139: 429-435, 2013.

43. de Vicente JC, Rosado P, Lequerica-Fernández P, Allonca E, Villallaín L and Hernández-Vallejo G: Focal adhesion kinase overexpression: Correlation with lymph node metastasis and shorter survival in oral squamous cell carcinoma. Head Neck 35: 826-830, 2013.

44. Cai HX, Yang LC, Song XH, Liu ZR, Chen YB and Dong GK: Expression of paxillin and FAK mRNA and the related clinical significance in esophageal carcinoma. Mol Med Rep 5: 469-472, 2012.

45. Park JH, Lee BL, Yoon J, Kim J, Kim MA, Yang HK and Kim WH: Focal adhesion kinase (FAK) gene amplification and its clinical implications in gastric cancer. Hum Pathol 41: 1664-1673, 2010.

46. Jiang H, Liu L, Ye J, Liu H, Xing S and Wu Y: Focal adhesion kinase serves as a marker of cervical lymph node metastasis and is a potential therapeutic target in tongue cancer. J Cancer Res Clin Oncol 136: 1295-1302, 2010.

47. Gabriel B, Hasenburg A, Waizenegger M, Orlowska-Volk M, Stickeler E and zur Hausen A: Expression of focal adhesion kinase in patients with endometrial cancer: a clinicopathologic study. Int J Gynecol Cancer 19: 1221-1225, 2009.

48. Rodrigo JP, Dominguez F, Suárez V, Canel M, Secades P and Chiara MD: Focal adhesion kinase and E-cadherin as markers for nodal metastasis in laryngeal cancer. Arch Otolaryngol Head Neck Surg 133: 145-150, 2007.

49. Miyazaki T, Kato H, Nakajima M, Sohda M, Fukai Y, Masuda N, Manda R, Fukuchi M, Tsukada K and Kuwano H: FAK overexpression is correlated with tumour invasiveness and lymph node metastasis in oesophageal squamous cell carcinoma. $\mathrm{Br}$ J Cancer 89: 140-145, 2003. 